\title{
Research Article \\ Optimality Conditions for Approximate Solutions in Multiobjective Optimization Problems
}

\author{
Ying Gao, ${ }^{1}$ Xinmin Yang, ${ }^{1}$ and Heung Wing Joseph Lee ${ }^{2}$ \\ ${ }^{1}$ Department of Mathematics, Chongqing Normal University, Chongqing 400047, China \\ 2 Department of Applied Mathematics, The Hong Kong Polytechnic University, \\ Hung Hom, Kowloon, Hong Kong \\ Correspondence should be addressed to Ying Gao, gaoyingimu@163.com
}

Received 18 July 2010; Accepted 25 October 2010

Academic Editor: Mohamed El-Gebeily

Copyright $@ 2010$ Ying Gao et al. This is an open access article distributed under the Creative Commons Attribution License, which permits unrestricted use, distribution, and reproduction in any medium, provided the original work is properly cited.

\begin{abstract}
We study first- and second-order necessary and sufficient optimality conditions for approximate (weakly, properly) efficient solutions of multiobjective optimization problems. Here, tangent cone, $\epsilon$-normal cone, cones of feasible directions, second-order tangent set, asymptotic second-order cone, and Hadamard upper (lower) directional derivatives are used in the characterizations. The results are first presented in convex cases and then generalized to nonconvex cases by employing local concepts.
\end{abstract}

\section{Introduction}

The investigation of the optimality conditions is one of the most attractive topics of optimization theory. For vector optimization, the optimality solutions can be characterized with the help of different geometrical concepts. Miettinen and Mäkelä [1] and Huang and Liu [2] derived several optimality conditions for efficient, weakly efficient, and properly efficient solutions of vector optimization problems with the help of several kinds of cones. Engau and Wiecek [3] derived the cone characterizations for approximate solutions of vector optimization problems by using translated cones. In [4], Aghezzaf and Hachimi obtained second-order optimality conditions by means of a second-order tangent set which can be considered an extension of the tangent cone; Cambini et al. [5] and Penot [6] introduced a new second-order tangent set called asymptotic second-order cone. Later, second-order optimality conditions for vector optimization problems have been widely studied by using these second-order tangent sets; see [7-9]. 
During the past decades, researchers and practitioners in optimization had a keen interest in approximate solutions of optimization problems. There are several important reasons for considering this kind of solutions. One of them is that an approximate solution of an optimization problem can be computed by using iterative algorithms or heuristic methods. In vector optimization, the notion of approximate solution has been defined in several ways. The first concept was introduced by Kutateladze [10] and has been used to establish vector variational principle, approximate Kuhn-Tucker-type conditions and approximate duality theorems, and so forth, (see [11-20]). Later, several authors have proposed other $\epsilon$-efficiency concepts (see, e.g., White [21]; Helbig [22] and Tanaka [23]).

In this paper, we derive different characterizations for approximate solutions by treating convex case and nonconvex cases. Giving up convexity naturally means that we need local instead of global analysis. Some definitions and notations are given in Section 2. In Section 3, we derive some characterizations for global approximate solutions of multiobjective optimization problems by using tangent cone, the cone of feasible directions and $\epsilon$-normal cone. Finally, in Section 3, we introduce some local approximate concepts and present some properties of these notions, and then, first and second-order sufficient conditions for local (properly) approximate efficient solutions of vector optimization problems are derived. These conditions are expressed by means of tangent cone, second-order tangent set and asymptotic second-order set. Finally, some sufficient conditions are given for local (weakly) approximate efficient solutions by using Hadamard upper (lower) directional derivatives.

\section{Preliminaries}

Let $R^{n}$ be the $n$-dimensional Euclidean space and let $R_{+}^{n}$ be its nonnegative orthant. Let $C$ be a subset of $R^{n}$, then, the cone generated by the set $C$ is defined as cone $(C)=\cup_{\alpha \geq 0} \alpha C$, and int $C$ and $\operatorname{cl} C$ referred to as the interior and the closure of the set $C$, respectively. A set $D \subset R^{n}$ is said to be a cone if cone $D=D$. We say that the cone $D$ is solid if int $D \neq \emptyset$, and pointed if $D \cap(-D) \subset\{0\}$. The cone $D$ is said to have a base $B$ if $B$ is convex, $0 \notin \operatorname{cl} B$ and $D=$ cone $B$. The positive polar cone and strict positive polar cone of $D$ are denoted by $D^{+}$ and $D^{s+}$, respectively.

Consider the following multiobjective optimization problem:

$$
\min \{f(x): x \in S\}
$$

where $S \subset R^{n}$ is an arbitrary nonempty set, $f: S \rightarrow R^{m}$. As usual, the preference relation $\leq$ defined in $R^{m}$ by a closed convex pointed cone $D \subset R^{m}$ is used, which models the preferences used by the decision-maker:

$$
y, z \in Y, \quad y \leq z \Longleftrightarrow y-z \in-D .
$$

We recall that $x_{0} \in S$ is an efficient solution of (2.1) with respect to $D$ if $\left(f\left(x_{0}\right)-D\right) \cap$ $f(S)=\left\{f\left(x_{0}\right)\right\} \cdot x_{0} \in S$ is a weakly efficient solution of (2.1) with respect to $D$ if $\left(f\left(x_{0}\right)-\right.$ int $D) \cap f(S)=\emptyset$ (in this case, it is assumed that $D$ is solid). $x_{0} \in S$ is a Benson properly efficient solution (see [24]) of (2.1) with respect to $D$ if cl cone $\left(f(S)+D-f\left(x_{0}\right)\right) \cap(-D)=\{0\}$. $x_{0} \in S$ is a Henig' properly efficient solution (see [24]) of (2.1) with respect to $D$ if $x_{0} \in$ $E\left(f, D^{\prime}\right)$, for some convex cone $D^{\prime}$ with $D \backslash\{0\} \subset$ int $D^{\prime}$. 
Definition 2.1 (see [18, 25]). Let $q \in D \backslash\{0\}$ be a fixed element, and $\epsilon \geq 0$.

(i) $x \in S$ is said to be a weakly $\epsilon q$-efficient solution of problem (2.1) if $(f(S)-f(x)+$ $\epsilon q) \cap(-\operatorname{int} D)=\emptyset$ (in this case it is assumed that $D$ is solid).

(ii) $x \in S$ is said to be a efficient $\epsilon q$-solution of problem (2.1) if $(f(S)-f(x)+\epsilon q) \cap(-D \backslash$ $\{0\})=\emptyset$.

(iii) $x \in S$ is said to be a properly $\epsilon q$-efficient solution of problem (2.1), if cl cone $(f(S)+$ $\epsilon q+D-f(x)) \cap(-D)=\{0\}$.

The sets of $\epsilon q$-efficient solutions, weakly $\epsilon q$-efficient solutions, and properly $\epsilon q$ efficient solutions of problem (2.1) are denoted by $\operatorname{AE}(f, S, \epsilon q), \operatorname{WAE}(f, S, \epsilon q)$, and $\operatorname{PAE}(f, S, \epsilon q)$, respectively.

Remark 2.2. If $\epsilon=0$, then $\epsilon q$-efficient solution, weakly $\epsilon q$-efficient solution, and properly $\epsilon q$ efficient solution reduce to efficient solution, weakly efficient solution and properly efficient solution of problem (2.1).

Definition 2.3. Let $Z \subset R^{m}$ be a nonempty convex set.

The contingent cone of $Z$ at $z \in Z$ is defined as

$$
T(z, Z)=\left\{d \in R^{m}: \text { there exists } t_{j} \downarrow 0 \text { and } d_{j} \longrightarrow d \text { such that } z+t_{j} d_{j} \in Z\right\} .
$$

The cone of feasible directions of $Z$ at $z \in Z$ is defined as

$$
F(z, Z)=\left\{d \in R^{m}: \text { there exists } t>0 \text { such that } z+t d \in Z\right\} .
$$

Let $\epsilon \geq 0$, the $\epsilon$-normal set of $Z$ at $z \in Z$ is defined as

$$
N_{\epsilon}(z, Z)=\left\{y \in R^{m}: y^{T}(x-z) \leq \epsilon, \forall x \in Z\right\}
$$

Lemma 2.4 (see [26]). Let $N, K \subset R^{m}$ be closed convex cones such that $N \cap K=\{0\}$. Suppose that $K$ is pointed and locally compact, or int $K^{+} \neq \emptyset$, then, $\left(-N^{+}\right) \cap K^{s+} \neq \emptyset$.

\section{Cone Characterizations of Approximate Solutions: Convex Case}

In this section, we assume that $f(S)$ is a convex set.

Theorem 3.1. Let $\bar{x} \in S$ and $\epsilon \geq 0$. If

$$
F(f(\bar{x}), f(S)) \cap(-\epsilon q-D \backslash\{0\})=\emptyset,
$$

then $\bar{x} \in A E(f, S, \epsilon q)$.

Proof. Suppose, on the contrary, that $\bar{x} \notin \mathrm{AE}(f, S, \epsilon q)$, then, there exist $x \in S$ and $p \in D \backslash\{0\}$ such that $f(x)-f(\bar{x})+\epsilon q=-p$. That is, $f(x)=f(\bar{x})+(-\epsilon q-p)$. Therefore, $-\epsilon q-p \in$ $F(f(\bar{x}), f(S))$, which is a contradiction to $F(f(\bar{x}), f(S)) \cap(-\epsilon q-D \backslash\{0\})=\emptyset$. This completes the proof. 
Theorem 3.2. Let $\bar{x} \in S$.

(i) If $T(f(\bar{x}), f(S)) \cap(-D \backslash\{0\})=\emptyset$, then $\bar{x} \in \operatorname{PAE}(f, S)$.

(ii) Let $\epsilon>0$, and $D$ is solid set and $q \in \operatorname{int} D$. If $T(f(\bar{x}), f(S)) \cap(-\epsilon q-D \backslash\{0\})=\emptyset$, then $\bar{x} \in \operatorname{PAE}(f, S, e q)$.

Proof. (i) Suppose, on the contrary, that $\bar{x} \notin \operatorname{PAE}(f, S)$, then, there exists $q \in-D \backslash\{0\}$ such that $q \in$ cl cone $(f(S)-f(\bar{x})+D)$. Hence, there exist $\lambda_{n} \in R_{+}, x_{n} \in S$ and $q_{n} \in D, n \in N$ such that $\lambda_{n}\left(f\left(x_{n}\right)-f(\bar{x})+q_{n}\right) \rightarrow q$. Since $q \neq 0$, there exists $n \in N$ such that $\lambda_{n}>0$.

Since $f(S)$ is convex set, cl cone $(f(S)-f(\bar{x}))=T(f(\bar{x}), f(S))$. Hence, cl cone $(f(S)-$ $f(\bar{x})) \cap(-D \backslash\{0\})=\emptyset$. From Lemma 2.4, there exists $u \in D^{s+}$ such that $\langle u, y\rangle \geq 0$, for all $y \in \operatorname{clcone}(f(S)-f(\bar{x}))$.

On the other hand, from $u \in D^{s+}$, we have $\langle u, q\rangle<0$. Therefore, there exists $n_{1} \in$ $N$ such that $\left\langle u, f\left(x_{n_{1}}\right)-f(\bar{x})+q_{n_{1}}\right\rangle<0$, and so $\left\langle u, f\left(x_{n_{1}}\right)-f(\bar{x})\right\rangle<0$, which deduces a contradiction, and the proof is completed.

(ii) Now, we let $\epsilon>0$. From $T(f(\bar{x}), f(S)) \cap(-\epsilon q-D \backslash\{0\})=\emptyset$, we have

$$
T(f(\bar{x}), f(S)) \cap(-\operatorname{int} D)=\emptyset .
$$

In fact, if there exists $p \in R^{m}$ such that $p \in T(f(\bar{x}), f(S)) \cap(-\operatorname{int} D)$, then, from $q \in \operatorname{int} D$ and $\epsilon>0$, there exists $\lambda>0$ such that $p_{1}=-\lambda p-\epsilon q \in D \backslash\{0\}$. Hence, $-\epsilon q-p_{1}=\lambda p \epsilon$ $T(f(\bar{x}), f(S)) \cap(-\epsilon q-D \backslash\{0\})$, which is a contradiction to the assumption.

Since $f(S)$ is a convex set, cl cone $(f(S)-f(\bar{x}))=T(f(\bar{x}), f(S))$. Hence,

$$
\text { cl cone }(f(S)-f(\bar{x})) \cap(-\operatorname{int} D)=\emptyset
$$

By using the convex separation theorem, there exists $u \in R^{m} \backslash\{0\}$ such that $\langle u, y\rangle \geq 0$, for all $y \in-\operatorname{int} D$ and $\langle u, y\rangle \leq 0$, for all $y \in \operatorname{cl} \operatorname{cone}(f(S)-f(\bar{x}))$. It is easy to get that $\langle u, y\rangle \geq 0$, for all $y \in-D$. Hence, $\langle u, y\rangle>0$, for all $y \in-\operatorname{int} D$.

Suppose, on the contrary, that $\bar{x} \notin \operatorname{PAE}(f, S, \in q)$, then, there exists $\bar{y} \in R^{m}$ such that

$$
\bar{y} \in \operatorname{clcone}(f(S)+\epsilon q+D-f(\bar{x})) \cap(-D \backslash\{0\}),
$$

and there exist $y_{n} \in \operatorname{cone}(f(S)+\epsilon q+D-f(\bar{x}))$, for all $n \in N$ such that $y_{n} \rightarrow \bar{y}$. That is, there exist $\lambda_{n} \geq 0, x_{n} \in S$ and $p_{n} \in D$, for all $n \in N$ such that $y_{n}=\lambda_{n}\left(f\left(x_{n}\right)+\epsilon q+p_{n}-f(\bar{x})\right)$, for all $n \in N$. Since $\bar{y} \neq 0$, there exists $n_{1} \in N$ such that $\lambda_{n}>0$, for all $n \geq n_{1}$. From $\epsilon>0, q \in \operatorname{int} D$ and $p_{n} \in D$, for all $n \in N$, we have $\epsilon q+p_{n} \in \operatorname{int} D$, for all $n \in N$. Therefore,

$$
\left\langle u, y_{n}\right\rangle=\lambda_{n}\left\{\left\langle u, f\left(x_{n}\right)-f(\bar{x})\right\rangle+\left\langle u, \epsilon q+p_{n}\right\rangle\right\}<\lambda_{n}\left\langle u, f\left(x_{n}\right)-f(\bar{x})\right\rangle \leq 0, \quad \forall n \geq n_{1} .
$$

Which implies $\langle u, \bar{y}\rangle<0$. On the other hand, from $\bar{y} \in-D \backslash\{0\}$, we have $\langle u, \bar{y}\rangle \geq 0$, which yields a contradiction. This completes the proof.

Remark 3.3. If $\epsilon=0$, then the conditions of Theorems 3.1 and 3.2 are also necessary(see [2]). But for $\epsilon>0$, these are not necessary conditions, see the following example. 
Example 3.4. Let $D=R_{+}^{2}, q=(1,1)^{T}, S=\left\{x \in R^{2}: x_{1} \geq 0, x_{2} \geq 0\right\}, f: S \rightarrow R^{2}, f(x)=x$, $\epsilon=1 / 2$ and $\bar{x}=(1 / 2,1 / 2)^{T}$, then, $\bar{x} \in \operatorname{AE}(f, S, \epsilon q)$ and $\bar{x} \in \operatorname{PAE}(f, S, \epsilon q)$. But $F(f(\bar{x}), f(S))=$ $R^{2}=T(f(\bar{x}), f(S))$. Hence, $F(f(\bar{x}), f(S)) \cap(-\epsilon q-D \backslash\{0\}) \neq \emptyset$ and $T(f(\bar{x}), f(S)) \cap(-\epsilon q-D \backslash$ $\{0\}) \neq \emptyset$.

Theorem 3.5. Let $\bar{x} \in S, \epsilon \geq 0, D$ be a solid set and $q \in \operatorname{int} D$. If there exists $u \in-\left(D^{+} \backslash\{0\}\right)$ such that $\langle-u, q\rangle \geq 1$ and $u \in N_{\epsilon}(f(\bar{x}), f(S))$, then $\bar{x} \in W A E(f, S, e q)$. Conversely, if $\bar{x} \in$ $W A E(f, S, \epsilon q)$, then there exists $u \in-\left(D^{+} \backslash\{0\}\right)$ such that $\langle-u, q\rangle=1$ and $u \in N_{\epsilon}(f(\bar{x}), f(S))$.

Proof. Assume that, there exists $u \in-\left(D^{+} \backslash\{0\}\right)$ such that $\langle-u, q\rangle \geq 1$ and $u \in N_{\epsilon}(f(\bar{x}), f(S))$. Suppose, on the contrary, that $\bar{x} \notin \operatorname{WAE}(f, S, \epsilon q)$, then, there exist $p \in-\operatorname{int} D$ and $x \in S$ such that $p=f(x)-f(\bar{x})+\epsilon q$. From $u \in-\left(D^{+} \backslash\{0\}\right)$ and $\langle-u, q\rangle \geq 1$, we have $\langle u, f(x)-f(\bar{x})+\epsilon q\rangle>0$. Hence,

$$
\langle u, f(x)-f(\bar{x})\rangle>-\langle u, \epsilon q\rangle \geq \epsilon .
$$

On the other hand, from $u \in N_{\epsilon}(f(\bar{x}), f(S))$, we have $\langle u, f(x)-f(\bar{x})\rangle \leq \epsilon$, which is a contradiction to the above inequality. Hence, $\bar{x} \in \operatorname{WAE}(f, S, \epsilon q)$.

Conversely, let $\bar{x} \in \operatorname{WAE}(f, S, \epsilon q)$, then, $(f(S)-f(\bar{x})+\epsilon q) \cap(-\operatorname{int} D)=\emptyset$. Since $f(S)$ is convex and $D$ is a convex cone, there exists $\bar{u} \in-\left(D^{+} \backslash\{0\}\right)$ such that $\left.\langle\bar{u}, f(x)-f(\bar{x})+\epsilon q)\right\rangle \leq$ 0 , for all $x \in S$. Since $q \in$ int $D$, there exists $u \in-\left(D^{+} \backslash\{0\}\right)$ such that $\langle-u, q\rangle=1$ and $\langle u, f(x)-f(\bar{x})+\epsilon q\rangle \leq 0$, for all $x \in S$. Therefore, $\langle u, f(x)-f(\bar{x})\rangle \leq-\langle u, \epsilon q\rangle=\epsilon$, for all $x \in S$, which implies $u \in N_{\epsilon}(f(\bar{x}), f(S))$. This completes the proof.

Theorem 3.6. Let $\bar{x} \in S$ and $\epsilon \geq 0$. If there exists $u \in-D^{s+}$ such that $\langle-u, q\rangle \geq 1$ and $u \in$ $N_{\epsilon}(f(\bar{x}), f(S))$, then $\bar{x} \in \operatorname{PAE}(f, S, \epsilon q)$. Conversely, assume that $D$ is a locally compact set, if $\bar{x} \in$ $\operatorname{PAE}(f, S, \epsilon q)$, then there exists $u \in-D^{s+}$ such that $\langle-u, q\rangle=1$ and $u \in N_{\epsilon}(f(\bar{x}), f(S))$.

Proof. Assume that, there exists $u \in-D^{s+}$ such that $\langle-u, q\rangle \geq 1$ and $u \in N_{\epsilon}(f(\bar{x}), f(S))$. Suppose, on the contrary, that $\bar{x} \notin \operatorname{PAE}(f, S, e q)$, then, there exists $p \in R^{m}$ such that

$$
p \in \operatorname{clcone}(f(S)+\epsilon q+D-f(\bar{x})) \cap(-D \backslash\{0\}),
$$

and there exists $p_{n} \in \operatorname{cone}(f(S)+\epsilon q+D-f(\bar{x}))$, for all $n \in N$ such that $p_{n} \rightarrow p$. From $u \in D^{s+}$ and $p \in(-D \backslash\{0\})$, we have $\langle u, p\rangle>0$. Hence, there exists $n_{1} \in N$ such that $\left\langle u, p_{n}\right\rangle>0$, for all $n \geq n_{1}$. From $p_{n} \in \operatorname{cone}(f(S)+\epsilon q+D-f(\bar{x}))$, for all $n \in N$, there exist $\lambda_{n} \geq 0, x_{n} \in S$, and $q_{n} \in D$ such that $p_{n}=\lambda_{n}\left(f\left(x_{n}\right)+\epsilon q+q_{n}-f(\bar{x})\right)$, for all $n \in N$. Therefore, $\left\langle u, f\left(x_{n}\right)+\epsilon q+q_{n}-f(\bar{x})\right\rangle>0$, for all $n \geq n_{1}$, which combing with $q_{n} \in D$ and $\langle-u, q\rangle \geq 1$ yields $\left\langle u, f\left(x_{n}\right)-f(\bar{x})\right\rangle>-\langle u, \epsilon q\rangle \geq \epsilon$, for all $n \geq n_{1}$, which is a contradiction to $u \in N_{\epsilon}(f(\bar{x}), f(S))$. Hence, $\bar{x} \in \operatorname{PAE}(f, S, \epsilon q)$.

Conversely, let $\bar{x} \in \operatorname{PAE}(f, S, \epsilon q)$, then,

$$
\text { cl cone }(f(S)+\epsilon q+D-f(\bar{x})) \cap(-D)=\{0\} .
$$

Since $f(S)$ is a convex set, clcone $(f(S)+\epsilon q+D-f(\bar{x}))$ is a closed convex cone. From Lemma 2.4, there exists $\bar{u} \in(-D)^{s+}=-D^{s+}$ such that $\bar{u} \in-(\operatorname{cl} \text { cone }(f(S)+\epsilon q+D-f(\bar{x})))^{+}$. Since $q \in \operatorname{int} D, D^{s+}$ and (cl cone $\left.(f(S)+\epsilon q+D-f(\bar{x}))\right)^{+}$are cone, there exists $u \in(-D)^{s+}$ such that $\langle-u, q\rangle=1$ and $u \in-(\operatorname{cl} \operatorname{cone}(f(S)+\epsilon q+D-f(\bar{x})))^{+}$. 
Now, we prove that $u \in N_{\epsilon}(f(\bar{x}), f(S))$. That is, $\langle u, f(x)-f(\bar{x})\rangle \leq \epsilon$, for all $x \in S$. From $u \in-(\operatorname{clcone}(f(S)+\epsilon q+D-f(\bar{x})))^{+}$, we have

$$
\langle u, f(x)-f(\bar{x})+\epsilon q+p\rangle \leq 0, \quad \forall x \in S, p \in D
$$

Since $0 \in D$ and $\langle-u, q\rangle=1$, we have

$$
\langle u, f(x)-f(\bar{x})\rangle \leq-\langle u, \epsilon q\rangle=\epsilon, \quad \forall x \in S
$$

Which implies $u \in N_{\epsilon}(f(\bar{x}), f(S))$. This completes the proof.

Example 3.7. Let $D=R_{+}^{2}, q=(1,1)^{T}, S=\left\{x \in R^{2}: x_{1} \geq 0, x_{2} \geq 0\right\}, f: S \rightarrow R^{2}, f(x)=x$, $\epsilon=1 / 2$ and $\bar{x}=(1 / 2,1 / 2)^{T}$, then, $\bar{x} \in \operatorname{WAE}(f, S, \epsilon)$ and $\bar{x} \in \operatorname{PAE}(f, S, \epsilon)$. Let $u=-(1 / 2,1 / 2)^{T}$, then $\langle u, p\rangle=1$ and $u \in N_{\epsilon}(f(\bar{x}), f(S))=\left\{x \in R^{2}: x_{1}+x_{2} \geq-1, x_{1} \leq 0, x_{0} \leq 0\right\}$.

Remark 3.8. (i) If $\epsilon=0$ and $D=R_{+}^{m}$, then Theorems 3.1 and 3.5 reduce to the corresponding results in [1].

(ii) In [1], the cone characterizations of Henig' properly efficient solution were derived. We know that Henig' properly efficient solution equivalent to Benson properly efficient solution, when $D$ is a closed convex pointed cone(see [24]). Therefore, if $\epsilon=0$ and $D=R_{+}^{m}$, Theorems 3.2 and 3.6 reduce to the corresponding results in [1].

\section{Cone Characterizations of Approximate Solutions: Nonconvex Case}

In this section, $f(S)$ is no longer assumed to be convex. In nonconvex case, the corresponding local concepts are defined as follows.

Definition 4.1. Let $q \in D \backslash\{0\}$ be a fixed element and $\epsilon \geq 0$.

(i) $x \in S$ is said to be a local weakly $\epsilon q$-efficient solution of problem (2.1), if there exists a neighborhood $V$ of $x$ such that $(f(S \cap V)-f(x)+\epsilon q) \cap(-$ int $D)=\emptyset$ (in this case, it is assumed that $D$ is solid).

(ii) $x \in S$ is said to be a local $\epsilon q$-efficient solution of problem (2.1), if there exists a neighborhood $V$ of $x$ such that $(f(S \cap V)-f(x)+\epsilon q) \cap(-D \backslash\{0\})=\emptyset$.

(iii) $x \in S$ is said to be a local properly $\epsilon q$-efficient solution of problem (2.1), if there exists a neighborhood $V$ of $x$ such that cl cone $(f(S \cap V)+\epsilon q+D-f(x)) \cap(-D)=\{0\}$.

The sets of local $\epsilon q$-efficient solutions, local weakly $\epsilon q$-efficient solutions and local properly $\epsilon q$-efficient solutions of problem (2.1) are denoted by $\operatorname{LAE}(f, S, \epsilon q), \operatorname{LWAE}(f, S, \epsilon q)$ and $\operatorname{LPAE}(f, S, \epsilon q)$, respectively.

If $\epsilon=0$, then, (i), (ii), and (iii) reduce to the definitions of local weakly efficient solution, local efficient solution and local properly efficient solution, respectively, and the sets of local (weakly, properly) efficient solutions of problem (2.1) are denoted by $\operatorname{LE}(f, S)(\operatorname{LWE}(f, S), \operatorname{LPE}(f, S))$, respectively. 
Definition 4.2 (see $[4,5])$. Let $Z \subset R^{m}$ and $y, v \in R^{m}$.

(i) The second-order tangent set to $Z$ at $(y, v)$ is defined as

$$
T^{2}(Z, y, v)=\left\{d \in R^{m}: \exists t_{n} \downarrow 0, \exists d_{n} \longrightarrow d \text { such that } y_{n}=y+t_{n} v+\frac{1}{2} t_{n}^{2} d_{n} \in Z, \forall n \in N\right\} .
$$

(ii) The asymptotic second-order tangent cone to $Z$ at $(y, v)$ is defined as

$$
\begin{aligned}
T^{\prime \prime}(Z, y, v)= & \left\{d \in R^{m}: \exists\left(t_{n}, r_{n}\right) \downarrow(0,0), \exists d_{n} \longrightarrow d\right. \\
& \text { such that } \left.\frac{t_{n}}{r_{n}} \longrightarrow 0, y_{n}=\bar{x}+t_{n} v+\frac{1}{2} t_{n} r_{n} d_{n} \in Z, \forall n \in N\right\} .
\end{aligned}
$$

In [4-9], some properties of second-order tangent sets have been derived, see the following Lemma.

Lemma 4.3. Let $y \in \mathrm{cl} Z$ and $v \in R^{m}$, then,

(i) $T^{2}(Z, y, v)$ and $T^{\prime \prime}(Z, y, v)$ are closed sets contained in cl cone [cone $\left.(Z-y)-v\right]$, and $T^{\prime \prime}(Z, y, v)$ is a cone.

(ii) If $v \notin T(y, Z)$, then $T^{2}(Z, y, v)=T^{\prime \prime}(Z, y, v)=\emptyset$. If $v \in T(y, Z)$, then $T^{2}(Z, y, v) \cup$ $T^{\prime \prime}(Z, y, v) \neq \emptyset$. If $y \in$ int $Z$, then $T^{2}(Z, y, v)=T^{\prime \prime}(Z, y, v)=R^{m}$, and $T^{2}(Z, y, 0)=$ $T^{\prime \prime}(Z, y, 0)=T(y, Z)$.

(iii) Let $Z$ is convex. If $v \in T(y, Z)$ and $T^{\prime \prime}(Z, y, v) \neq \emptyset$, then $T^{2}(Z, y, v) \subset T^{\prime \prime}(Z, y, v)=$ cl cone $[\operatorname{cone}(Z-y)-v]=T(v, T(Z, y))$.

Definition 4.4 (see [27]). Let $K \subset R^{n}$ and $\phi: K \rightarrow R$ be a nonsmooth function. The Hadamard upper directional derivative and the Hadamard lower directional derivative derivative of $\phi$ at $x \in K$ in the direction $d \in R^{n}$ are given by

$$
\begin{aligned}
& \phi_{+}^{\prime}(x, d)=\lim _{t \downarrow 0} \sup _{h \rightarrow d} \frac{\phi(x+t h)-\phi(x)}{t}, \\
& \phi_{-}^{\prime}(x, d)=\lim _{t \downarrow 0} \inf _{h \rightarrow d} \frac{\phi(x+t h)-\phi(x)}{t} .
\end{aligned}
$$

Lemma 4.5 (see [7]). Let $Y$ be a finite-dimensional space and $y_{0} \in E \subset Y$. If the sequence $y_{n} \in$ $E \backslash\left\{y_{0}\right\}$ converges to $y_{0}$, then there exists a subsequence (denoted the same) $y_{n}$ such that $\left(y_{n}-\right.$ $\left.y_{0}\right) / t_{n}$ converges to some nonnull vector $u \in T\left(y_{0}, E\right)$, where $t_{n}=\left\|y_{n}-y_{0}\right\|$, and either $\left(y_{n}-y_{0}-\right.$ $\left.t_{n} u\right) /(1 / 2) t_{n}^{2}$ converges to some vector $z \in T^{2}\left(E, y_{0}, u\right) \cap u^{\perp}$ or there exists a sequence $r_{n} \rightarrow 0^{+}$such that $t_{n} / r_{n} \rightarrow 0$ and $\left(y_{n}-y_{0}-t_{n} u\right) /(1 / 2) t_{n} r_{n}$ converges to some vector $z \in T^{\prime \prime}\left(E, y_{0}, u\right) \cap u^{\perp} \backslash\{0\}$, where $u^{\perp}$ denotes the orthogonal subspace to $u$.

In the following theorem, we derive several properties of local (weakly, properly) approximate efficient solutions. 
Theorem 4.6. (i) Let int $D \neq \emptyset$, then, for any fixed $q \in D \backslash\{0\}$,

$$
\operatorname{LWE}(f, S) \subset \bigcap_{\epsilon>0} \operatorname{LWAE}(f, S, \epsilon q) .
$$

Conversely, if $\bar{x} \in S$, and there exists a neighborhood $\bar{V}$ of $\bar{x}$ such that $(f(S \cap \bar{V})-f(\bar{x})) \cap(-\epsilon q-$ $\operatorname{int} D)=\emptyset$, for all $\epsilon>0$, that is, $\bar{x} \in W A E(f, S \cap \bar{V}, \epsilon q)$, for all $\epsilon>0$, then $\bar{x} \in \operatorname{LWE}(f, S)$.

(ii) For any fixed $q \in D \backslash\{0\}, L E(f, S) \subset \bigcap_{e>0} L A E(f, S, e q)$. Conversely, if $\bar{x} \in S$ and there exists a neighborhood $\bar{V}$ of $\bar{x}$ such that for any fixed $q \in D \backslash\{0\}$ and $\epsilon>0,(f(S \cap \bar{V})-f(\bar{x})) \cap$ $(-\epsilon q-D \backslash\{0\})=\emptyset$, then $\bar{x} \in L E(f, S)$.

(iii) For any fixed $q \in D \backslash\{0\}, \operatorname{LPE}(f, S) \subset \bigcap_{e>0} \operatorname{LPAE}(f, S, \epsilon q)$. Conversely, if $\bar{x} \in S$ and there exists a neighborhood $\bar{V}$ of $\bar{x}$ such that for any fixed $q \in D \backslash\{0\}$ and $\epsilon>0$, cone $(f(S \cap$ $\bar{V})-f(\bar{x})+\epsilon q+D)$ is a closed set, and (cl cone $(f(S \cap \bar{V})-f(\bar{x})+\epsilon q+D)) \cap(-D)=\{0\}$, then $\bar{x} \in \operatorname{LPE}(f, S)$.

Proof. (i) Let $\bar{x} \in \operatorname{LWE}(f, S)$, then, there exists a neighborhood $V_{1}$ of $\bar{x}$ such that $f\left(S \cap V_{1}\right)$ $f(\bar{x}) \cap(-\operatorname{int} D)=\emptyset$. From $q \in D \backslash\{0\}$, we have

$$
f\left(S \cap V_{1}\right)-f(\bar{x}) \cap(-\epsilon q-\operatorname{int} D)=\emptyset, \quad \forall \epsilon>0 .
$$

Which implies $\bar{x} \in \bigcap_{\epsilon>0} \operatorname{LWAE}(f, S, \epsilon q)$.

Conversely, we assume that there exists a neighborhood $\bar{V}$ of $\bar{x}$ such that $\bar{x} \in$ $\operatorname{WAE}(f, S \cap \bar{V}, \epsilon q)$, for all $\epsilon>0$. Suppose, on the contrary, that $\bar{x} \notin \operatorname{LWE}(f, S)$, then, for any neighborhood $V$ of $\bar{x} f(S \cap V)-f(\bar{x}) \cap(-\operatorname{int} D) \neq \emptyset$. Take $V=\bar{V}$, then, there exist $p \in \operatorname{int} D$ and $x \in S \cap \bar{V}$ such that $f(x)-f(\bar{x})=-p$. Therefore, if $\epsilon>0$ is sufficiently small, we have $f(x)-f(\bar{x})=-p=-\epsilon q-(p-\epsilon q) \in-\epsilon q-\operatorname{int} D$, which is a contradiction to $\bar{x} \in \operatorname{WAE}(f, S \cap \bar{V}, \epsilon q)$, for all $\epsilon>0$. This completes the proof.

(ii) It is easy to see that $\operatorname{LE}(f, S) \subset \bigcap_{e>0} \operatorname{LAE}(f, S, \epsilon q)$.

Conversely, we assume that there exists a neighborhood $\bar{V}$ of $\bar{x}$ such that for any fixed $q \in D \backslash\{0\}$ and $\epsilon>0,(f(S \cap \bar{V})-f(\bar{x})) \cap(-\bar{\epsilon} q-D \backslash\{0\})=\emptyset$. Suppose, on the contrary, that $\bar{x} \notin \mathrm{LE}(f, S)$, then, for any neighborhood $V$ of $\bar{x}$, we have $f(S \cap V)-f(\bar{x}) \cap(-D \backslash\{0\}) \neq \emptyset$. Take $V=\bar{V}$, then, there exist $\bar{p} \in D \backslash\{0\}$ and $x \in S \cap \bar{V}$ such that $f(x)-f(\bar{x})=-\bar{p}$. Take $q=\bar{p} / 2$ and $\epsilon=1$, then, $f(x)-f(\bar{x})=-\bar{p}=-\epsilon q-\bar{p} / 2 \in-\epsilon q-D \backslash\{0\}$, which is a contradiction to the assumption. This completes the proof.

(iii) It is easy to see that $\operatorname{LPE}(f, S) \subset \bigcap_{\epsilon>0} \operatorname{LPAE}(f, S, \epsilon q)$.

Conversely, we assume that there exists a neighborhood $\bar{V}$ of $\bar{x}$ such that for any fixed $q \in D \backslash\{0\}$ and $\epsilon>0$, cone $(f(S \cap \bar{V})-f(\bar{x})+\epsilon q+D)$ is a closed set, and (clcone $(f(S \cap$ $\bar{V})-f(\bar{x})+\epsilon q+D)) \cap(-D)=\{0\}$. Suppose, on the contrary, that $\bar{x} \notin \operatorname{LPE}(f, S)$, then, for any neighborhood $V$ of $\bar{x}$, we have cl cone $(f(S \cap V)-f(\bar{x})+D) \cap(-D \backslash\{0\}) \neq \emptyset$. Take $V=\bar{V}$, then, there exist $\lambda>0, \bar{p}_{1} \in D \backslash\{0\}, \bar{p}_{2} \in D$ and $x \in S \cap \bar{V}$ such that $\lambda\left(f(x)-f(\bar{x})+\bar{p}_{2}\right)=-\bar{p}_{1}$. Take $q=p_{1} / 2 \lambda$ and $\epsilon=1$, similar to the proof of (ii) we can complete the proof. 
Theorem 4.7. Let $f$ be a continuous function on $S, \bar{x} \in S$, and $\epsilon>0$.

(i) If $T(f(\bar{x}), f(S)) \cap(-\epsilon q-D)=\emptyset$, then $\bar{x} \in \operatorname{LAE}(f, S, \epsilon q)$.

(ii) If $T(f(\bar{x}), f(S)) \cap(-\epsilon q-D) \neq \emptyset$, and for each $v \in T(f(\bar{x}), f(S)) \cap(-\epsilon q-D)$

$$
\begin{gathered}
T^{2}(f(S), f(\bar{x}), v) \cap v^{\perp} \cap(- \text { cl cone }(D+\epsilon q+v))=\emptyset, \\
T^{\prime \prime}(f(S), f(\bar{x}), v) \cap v^{\perp} \cap(-\operatorname{clcone}(D+\epsilon q+v))=\{0\}
\end{gathered}
$$

then $\bar{x} \in \operatorname{LAE}(f, S, \epsilon q)$.

Proof. (i) Let $T(f(\bar{x}), f(S)) \cap(-\epsilon q-D)=\emptyset$. Suppose, on the contrary, that $\bar{x} \notin \operatorname{LAE}(f, S, \epsilon q)$, then, there exists $x_{n} \in S$ and $x_{n} \rightarrow \bar{x}$ such that $f\left(x_{n}\right)-f(\bar{x})+\epsilon q \in-D \backslash\{0\}$, for all $n \in N$. Since $f$ is a continuous function and $D$ is a pointed cone, $f\left(x_{n}\right) \neq f(\bar{x})$, for all $n \in N$ and $f\left(x_{n}\right) \rightarrow f(\bar{x})$. Therefore, $\left(f\left(x_{n}\right)-f(\bar{x})\right) /\left\|f\left(x_{n}\right)-f(\bar{x})\right\| \rightarrow d \in T(f(\bar{x}), f(S))$.

On the other hand, for any $n \in N$, we have

$$
\begin{aligned}
\frac{f\left(x_{n}\right)-f(\bar{x})}{\left\|f\left(x_{n}\right)-f(\bar{x})\right\|} & \in-\frac{1}{\left\|f\left(x_{n}\right)-f(\bar{x})\right\|}(\epsilon q+D \backslash\{0\}) \\
& \subset-\left(\epsilon q+D \backslash\{0\}+\left(\frac{1}{\left\|f\left(x_{n}\right)-f(\bar{x})\right\|}-1\right) \epsilon q\right) .
\end{aligned}
$$

Since $f\left(x_{n}\right) \rightarrow f(\bar{x})$ and $q \in D \backslash\{0\}$, there exists $n_{1} \in N$ such that

$$
\left(\frac{1}{\left\|f\left(x_{n}\right)-f(\bar{x})\right\|}-1\right) \epsilon q \in D, \quad \forall n \geq n_{1} .
$$

Hence, $d \in-(e q+D)$, which is a contradiction to the assumption. This completes the proof.

(ii) Suppose, on the contrary, that $\bar{x} \notin \operatorname{LAE}(f, S, \epsilon q)$. Similar to the proof of (i), we have there exists $x_{n} \rightarrow \bar{x}$ such that

$$
\frac{f\left(x_{n}\right)-f(\bar{x})}{\left\|f\left(x_{n}\right)-f(\bar{x})\right\|} \longrightarrow d \in T(f(\bar{x}), f(S)) \cap(-\epsilon q-D)
$$

Let $t_{n}=\left\|f\left(x_{n}\right)-f(\bar{x})\right\|$ and $z_{n}=\left(2 / t_{n}\right)\left(\left(f\left(x_{n}\right)-f(\bar{x})\right) / t_{n}-d\right)$, for all $n \in N$. Similar to the proof of Lemma 4.3, we have there exists $z \in R^{m}$ such that $z \in T^{2}(f(S), f(\bar{x}), d) \cap d^{\perp} \cap$ -cl cone $(\epsilon q+D+d)$ or $z \in T^{\prime \prime}(f(S), f(\bar{x}), d) \cap d^{\perp} \backslash\{0\} \cap$-cl cone $(\epsilon q+D+d)$, which is a contradiction to the assumptions. This completes the proof. 
Corollary 4.8. Let $f$ be a continuous function on $S, \bar{x} \in S$ and $\epsilon=0$.

(i) If $T(f(\bar{x}), f(S)) \cap(-D)=\{0\}$, then $\bar{x}$ is a local efficient solution of problem (2.1).

(ii) If $T(f(\bar{x}), f(S)) \cap(-D \backslash\{0\}) \neq \emptyset$, and for each $v \in T(f(\bar{x}), f(S)) \cap(-D \backslash\{0\})$

$$
\begin{gathered}
T^{2}(f(S), f(\bar{x}), v) \cap v^{\perp} \cap(- \text { cl cone }(D+v))=\emptyset, \\
T^{\prime \prime}(f(S), f(\bar{x}), v) \cap v^{\perp} \cap(- \text { cl cone }(D+v))=\{0\},
\end{gathered}
$$

then $\bar{x}$ is a local efficient solution of problem (2.1).

Proof. The proof is similar to Theorem 4.7 .

Remark 4.9. If $f(S)$ is convex, then the condition (ii) of Theorem 4.7 is equivalent to the following condition

(ii) $^{\prime} T(f(\bar{x}), f(S)) \cap(-\epsilon q-D) \neq \emptyset$, and for each $v \in T(f(\bar{x}), f(S)) \cap(-\epsilon q-D)$

$$
0 \notin T^{2}(f(S), f(\bar{x}), v), \quad T^{\prime \prime}(f(S), f(\bar{x}), v) \cap v^{\perp} \cap(- \text { cl cone }(D+\epsilon q+v))=\{0\},
$$

since $T^{2}(f(S), f(\bar{x}), v) \subset T^{\prime \prime}(f(S), f(\bar{x}), v)$ by Lemma 4.3(iii).

Theorem 4.10. Let $f$ be continuous on $S, \bar{x} \in S$, and $\epsilon \geq 0$.

(i) Assume that $D$ has a compact base $B, p=\alpha b$ for $b \in B$ and $\alpha>0$, and there exists $\delta>0$ such that $(f(S)-f(\bar{x})) \cap \delta U \subset T(f(\bar{x}), f(S))$. If $T(f(\bar{x}), f(S)) \cap(-\epsilon q-D \backslash\{0\})=\emptyset$, then $\bar{x} \in \operatorname{LPAE}(f, S, \epsilon q)$.

(ii) Assume that $T(f(\bar{x}), f(S)) \cap(-\epsilon q-D \backslash\{0\}) \neq \emptyset$, and there exists $\beta>0$ such that for each $d \in(T(f(\bar{x}), f(S)) \backslash\{0\}) \cap(-\epsilon q-D+\beta U)$ the following conditions hold

$$
\begin{aligned}
& T^{2}(f(S), f(\bar{x}), d) \cap d^{\perp} \cap(-\operatorname{clcone}(D+\epsilon q+\beta U+d))=\emptyset, \\
& T^{\prime \prime}(f(S), f(\bar{x}), d) \cap d^{\perp} \cap(-\operatorname{clcone}(D+\epsilon q+\beta U+d))=\{0\}
\end{aligned}
$$

then $\bar{x} \in \operatorname{LPAE}(f, S, \epsilon q)$, where, $U$ denotes the closed unit ball of $R^{m}$.

Proof. (i) Let $T(f(\bar{x}), f(S)) \cap(-\epsilon q-D \backslash\{0\})=\emptyset$, then, $T(f(\bar{x}), f(S)) \cap(-\lambda \epsilon b-B)=\emptyset$, for all $\lambda>0$. The assumptions and the separation result [28, page 9] implies that for any $\lambda>0$ there exists a neighborhood $V_{\lambda}$ of 0 such that

$$
T(f(\bar{x}), f(S)) \cap\left(-\lambda \epsilon b-B+V_{\curlywedge}\right)=\emptyset .
$$

Suppose, on the contrary, that $\bar{x} \notin \operatorname{LPAE}(f, S, \epsilon q)$, then, for any neighborhood $V$ of 0 , we have

$$
\text { cl cone }\left(f\left(S \cap\left(\bar{x}+\frac{V}{n}\right)\right)-f(\bar{x})+\epsilon q+D\right) \cap(-D \backslash\{0\}) \neq \emptyset
$$


Therefore,

$$
\text { cl cone }\left(f\left(S \cap\left(\bar{x}+\frac{V}{n}\right)\right)-f(\bar{x})+\epsilon q+D\right) \cap-B \neq \emptyset
$$

That is, for any $n \in N$ there exist $z_{n} \in \operatorname{cl} \operatorname{cone}(f(S \cap(\bar{x}+V / n))-f(\bar{x})+\epsilon q+D) \cap(-B)$, and so, for any $n \in N$ there exists $\lambda_{n}^{k} \geq 0, x_{n}^{k} \in S \cap(\bar{x}+V / n)$ and $p_{n}^{k} \in D$ such that $z_{n}^{k}=$ $\lambda_{n}^{k}\left(f\left(x_{n}^{k}\right)-f(\bar{x})+\epsilon q+p_{n}^{k}\right)$ and $z_{n}^{k} \rightarrow z_{n}$. Since $z_{n}^{k} \rightarrow z_{n}$, there exists $k_{1} \in N$ such that $z_{n}^{k} \in z_{n}+V$, for all $k \geq k_{1}$. By $z_{n}^{k}=\lambda_{n}^{k}\left(f\left(x_{n}^{k}\right)-f(\bar{x})+\epsilon q+p_{n}^{k}\right)$, we have

$$
\lambda_{n}^{k}\left(f\left(x_{n}^{k}\right)-f(\bar{x})\right) \in z_{n}+V-\lambda_{n}^{k}\left(\epsilon q+p_{n}^{k}\right), \quad \forall k \geq k_{1} .
$$

Let $p_{n}^{k}=\beta_{n}^{k} \theta_{n}^{k}$ for $\beta_{n}^{k} \geq 0$ and $\theta_{n}^{k} \in B$, then,

$$
\frac{\lambda_{n}^{k}}{1+\lambda_{n}^{k} \beta_{n}^{k}}\left(f\left(x_{n}^{k}\right)-f(\bar{x})\right) \in-\left(-\frac{z_{n}}{1+\lambda_{n}^{k} \beta_{n}^{k}}+\frac{\lambda_{n}^{k} \beta_{n}^{k} \theta_{n}^{k}}{1+\lambda_{n}^{k} \beta_{n}^{k}}\right)-\frac{\alpha \epsilon \lambda_{n}^{k} b}{1+\lambda_{n}^{k} \beta_{n}^{k}}+\frac{V}{1+\lambda_{n}^{k} \beta_{n}^{k}} .
$$

Let $\gamma_{n}^{k}=-z_{n} /\left(1+\lambda_{n}^{k} \beta_{n}^{k}\right)+\lambda_{n}^{k} \beta_{n}^{k} \theta_{n}^{k} /\left(1+\lambda_{n}^{k} \beta_{n}^{k}\right)$, then, $\gamma_{n}^{k} \in B$, since $B$ is a convex set, and so,

$$
\frac{\lambda_{n}^{k}}{1+\lambda_{n}^{k} \beta_{n}^{k}}\left(f\left(x_{n}^{k}\right)-f(\bar{x})\right) \in-\gamma_{n}^{k}-\frac{\alpha \epsilon \lambda_{n}^{k} b}{1+\lambda_{n}^{k} \beta_{n}^{k}}+\frac{V}{1+\lambda_{n}^{k} \beta_{n}^{k}}, \quad \forall k \geq k_{1} .
$$

On the other hand, from $x_{n}^{k} \in S \cap(\bar{x}+V / n)$, we have $x_{n}^{k} \rightarrow \bar{x}$ when $n \rightarrow \infty$ and $k \rightarrow \infty$. Since $f$ is a continuous function, $f\left(x_{n}^{k}\right) \rightarrow f(\bar{x})$ when $n \rightarrow \infty$ and $k \rightarrow \infty$, which combining with the assumption $(f(S)-f(\bar{x})) \cap \delta U \subset T(f(\bar{x}), f(S))$ yields there exist $n_{1} \in N$ and $k_{n_{1}} \in N$ such that

$$
f\left(x_{n_{1}}^{k}\right)-f(\bar{x}) \in(f(S)-f(\bar{x})) \cap \delta U \subset T(f(\bar{x}), f(S)), \quad \forall k \geq k_{n_{1}} .
$$

From $z_{n_{1}} \neq 0$, there exists $\bar{k}_{n_{1}} \in N$ such that $\lambda_{n_{1}}^{k}>0$, for all $k \geq \bar{k}_{n_{1}}$. Take $k_{2}=\max \left\{k_{n_{1}}, \bar{k}_{n_{1}}\right\}$, and let $\lambda=\alpha \lambda_{n_{1}}^{k_{2}} /\left(1+\lambda_{n_{1}}^{k_{2}} \beta_{n_{1}}^{k_{2}}\right)>0$. Since $V$ is an arbitrary set, it follows that

$$
\frac{\lambda_{n_{1}}^{k_{2}}}{1+\lambda_{n_{1}}^{k_{2}} \beta_{n_{1}}^{k_{2}}}\left(f\left(x_{n_{1}}^{k_{2}}\right)-f(\bar{x})\right) \in\left(-B-\lambda \epsilon b+V_{\curlywedge}\right)
$$

Which is a contradiction to (4.13). This completes the proof.

(ii) Suppose, on the contrary, that $\bar{x} \notin \operatorname{LPAE}(f, S, \epsilon)$, then, for any $r>0$ and $n \in N$, we have

$$
\text { cl cone }\left(f\left(S \cap\left(\bar{x}+\frac{\gamma U}{n}\right)\right)-f(\bar{x})+\epsilon q+D\right) \cap(-D \backslash\{0\}) \neq \emptyset
$$

Let $V=\gamma U$. Similar to the proof of (i), we have for any $n \in N$ there exist $\lambda_{n}^{k} \geq 0, x_{n}^{k} \in$ $S \cap(\bar{x}+V / n)$, and $p_{n}^{k} \in D$ such that $z_{n}^{k}=\lambda_{n}^{k}\left(f\left(x_{n}^{k}\right)-f(\bar{x})+\epsilon q+p_{n}^{k}\right)$ and $z_{n}^{k} \rightarrow z_{n}$. It is 
obvious that $f\left(x_{n}^{k}\right) \neq f(\bar{x})$. Otherwise, $z_{n} \in(e q+D) \cap(-D \backslash\{0\})$, which is a contradiction to the assumption that $D$ is a pointed cone. Since $z_{n} \neq 0$ and $z_{n}^{k} \rightarrow z_{n}$, there exists $k_{1} \in N$ such that $\lambda_{n}^{k}>0$ and $z_{n}^{k} \in z_{n}+V$, for all $k \geq k_{1}$. From $x_{n}^{k} \in S \cap(\bar{x}+V / n)$, we have $x_{n}^{k} \rightarrow \bar{x}$, when $n \rightarrow \infty$ and $k \rightarrow \infty$. Since $f$ is a continuous function and $f\left(x_{n}^{k}\right) \neq f(\bar{x})$, it is easy to see that $\left(f\left(x_{n}^{k}\right)-f(\bar{x})\right) /\left\|f\left(x_{n}^{k}\right)-f(\bar{x})\right\| \rightarrow d \in T(f(\bar{x}), f(S))$. From $z_{n}^{k}=\lambda_{n}^{k}\left(f\left(x_{n}^{k}\right)-f(\bar{x})+\epsilon q+p_{n}^{k}\right)$, we have for sufficiently large $n, k \in N$

$$
\frac{f\left(x_{n}^{k}\right)-f(\bar{x})}{\left\|f\left(x_{n}^{k}\right)-f(\bar{x})\right\|}=\frac{z_{n}^{k}-\lambda_{n}^{k}\left(\epsilon q+p_{n}^{k}\right)}{\lambda_{n}^{k}\left\|f\left(x_{n}^{k}\right)-f(\bar{x})\right\|}
$$

On the other hand, we have

$$
\frac{z_{n}^{k}-\lambda_{n}^{k}\left(\epsilon q+p_{n}^{k}\right)}{\lambda_{n}^{k}\left\|f\left(x_{n}^{k}\right)-f(\bar{x})\right\|} \in-\epsilon q-D+V
$$

for sufficiently large $k, n \in N$. In fact, for sufficiently large $k, n \in N$

$$
\frac{z_{n}^{k}-\lambda_{n}^{k}\left(\epsilon q+p_{n}^{k}\right)}{\lambda_{n}^{k}\left\|f\left(x_{n}^{k}\right)-f(\bar{x})\right\|} \in \frac{z_{n}+V-\lambda_{n}^{k}(\epsilon q+D)}{\lambda_{n}^{k}\left\|f\left(x_{n}^{k}\right)-f(\bar{x})\right\|}
$$

Hence,

$$
\frac{z_{n}^{k}-\lambda_{n}^{k}\left(\epsilon q+p_{n}^{k}\right)}{\lambda_{n}^{k}\left\|f\left(x_{n}^{k}\right)-f(\bar{x})\right\|} \in-\epsilon q-D+\frac{V}{\lambda_{n}^{k}\left\|f\left(x_{n}^{k}\right)-f(\bar{x})\right\|}
$$

when $k$ and $n$ sufficiently large enough. Since $\gamma>0$ is arbitrary,

$$
\frac{f\left(x_{n}^{k}\right)-f(\bar{x})}{\left\|f\left(x_{n}^{k}\right)-f(\bar{x})\right\|}=\frac{z_{n}^{k}-\lambda_{n}^{k}\left(\epsilon q+p_{n}^{k}\right)}{\lambda_{n}^{k}\left\|f\left(x_{n}^{k}\right)-f(\bar{x})\right\|} \longrightarrow d \in(-\epsilon q-D+\beta U) \cap T(f(\bar{x}), f(S)) .
$$

Let $t_{n}^{k}=\left\|f\left(x_{n}^{k}\right)-f(\bar{x})\right\|$ and $z_{n}^{k}=\left(2 / t_{n}^{k}\right)\left(\left(f\left(x_{n}^{k}\right)-f(\bar{x})\right) / t_{n}^{k}-d\right)$. Similar to the proof of Lemma 4.3, we have there exists $z \in R^{m}$ such that $z \in T^{2}(f(S), f(\bar{x}), d) \cap d^{\perp} \cap-$ cl cone $(\epsilon q+D+$ $d+\beta U)$ or $z \in T^{\prime \prime}(f(S), f(\bar{x}), d) \cap d^{\perp} \backslash\{0\} \cap$-cl cone $(\epsilon q+D+d+\beta U)$, which is a contradiction to the assumptions. This completes the proof. 
Remark 4.11. If $f(S)$ is convex, then the conditions (i) and (ii) of Theorem 4.10 are equivalent to (i)' and (ii)", respectively.

(i) ${ }^{\prime} D$ has a compact base $B, p=\alpha b$ for some $b \in B, \alpha>0$, and $T(f(\bar{x}), f(S)) \cap(-\epsilon q-$ $D \backslash\{0\})=\emptyset$.

(ii) $T(f(\bar{x}), f(S)) \cap(-\epsilon q-D \backslash\{0\}) \neq \emptyset$, and there exists $\beta>0$ such that for each $d \in$ $(T(f(\bar{x}), f(S)) \backslash\{0\}) \cap(-\epsilon q-D+\beta U)$

$$
0 \notin T^{2}(f(S), f(\bar{x}), d), \quad T^{\prime \prime}(f(S), f(\bar{x}), d) \cap d^{\perp} \cap(- \text { cl cone }(D+\epsilon q+\beta U+d))=\{0\} .
$$

Remark 4.12. The conditions of Theorem 4.7, Corollary 4.8 and Theorem 4.10 are not necessary conditions, see Examples 4.14 and 4.15.

Now, we give some examples to verify the results of Theorem 4.7, Theorem 4.10 and Corollary 4.8.

Example 4.13. Let $D=R_{+}^{2}, S=\left\{\left(x_{1}, x_{2}\right) \in R^{2}: x_{2} \geq\left|x_{1}\right|^{3 / 2}\right\}, f: S \rightarrow R^{2}, f\left(x_{1}, x_{2}\right)=$ $\left(x_{1}, x_{2}\right)^{T}, q=(1,1)^{T}$, and $\epsilon>0$. We consider $\bar{x}=(0,0)^{T} \in S$. It is easy to see that $T(f(\bar{x}), f(S)) \cap(-\epsilon q-D)=\emptyset$ and $f(S)-f(\bar{x}) \subset T(f(\bar{x}), f(S))$. That is, the condition (i) of Theorem 4.10 is valid, and $\bar{x} \in \operatorname{LPAE}(f, S, \epsilon q)=\operatorname{PAE}(f, S, \epsilon q)$, for all $\epsilon>0$.

If we let $0<\epsilon<1$ and $\bar{x}=\left(\epsilon, \epsilon^{3 / 2}\right)^{T} \in S$, then, $T(f(\bar{x}), f(S)) \cap(-\epsilon q-D) \neq \emptyset$. But the condition (ii) of Theorem 4.10 is valid. Hence, $\bar{x} \in \operatorname{LPAE}(f, S, \epsilon)=\operatorname{PEA}(f, S, \epsilon)$.

Let $\epsilon=0$, then, $T(f(\bar{x}), f(S)) \cap(-D \backslash\{0\}) \neq \emptyset$. But for all $d \in T(f(\bar{x}), f(S)) \cap(-D \backslash\{0\})$, the condition (ii) of Corollary 4.8 satisfies (see Example 3.7 in [7]), and $\bar{x}$ is an efficient solution of this problem, since $f(S)$ is a convex set. But for any $\beta>0$, it is easy to check that there exists $d \in(T(f(\bar{x}), f(S)) \backslash\{0\}) \cap(-D+\beta U)$ such that $T^{\prime \prime}(f(S), f(\bar{x}), d)=$ $T^{2}(f(S), f(\bar{x}), d)=$ cl cone $(D+d+\beta U)=R^{2}$. In fact, for any $\beta>0$, take $d=(\beta / 2, \beta / 2)^{T} \in$ $(T(f(\bar{x}), f(S)) \backslash\{0\}) \cap(-D+\beta U)$, then, $T^{\prime \prime}(f(S), f(\bar{x}), d)=T^{2}(f(S), f(\bar{x}), d)=$ cl cone $(D+d+$ $\beta U)=R^{2}$ and $d^{\perp}=\left\{y=\left(y_{1}, y_{2}\right)^{T} \in R^{2}: y_{1}+y_{2}=0\right\}$. Hence, the condition (ii) of Theorem 4.10 is false, and $\bar{x}$ is not a properly efficient solution of this problem.

Example 4.14. Let $D=R_{+}^{2} q=(1,1)^{T}, S=\left\{\left(x_{1}, x_{2}\right)^{T}: x_{1}+x_{2} \geq 0\right\} \cup\left\{\left(x_{1}, x_{2}\right) \in R^{2}: x_{1} \geq\right.$ $1\} \cup\left\{\left(x_{1}, x_{2}\right) \in R^{2}: x_{2} \geq 1\right\}, f(x): S \rightarrow R^{2}$ and $f(x)=x$. Take $\bar{x}=(0,0)^{T}$, then, it is easy to see that there exists $\delta>0$ such that $(f(S)-f(\bar{x})) \cap \delta U \subset T(f(\bar{x}) . f(S))$ and $T(f(\bar{x}), f(S)) \cap$ $(-\epsilon q-D \backslash\{0\})=\emptyset$, for all $\epsilon \geq 0$. Hence, $\bar{x} \in \operatorname{LPAE}(f, \epsilon, p)$, for all $\epsilon \geq 0$. But $\bar{x}$ is not a global properly efficient solution, where, $U$ is closed unit ball of $R^{2}$.

We let $0<\epsilon<1$ and $\bar{x}=(\epsilon, \epsilon)^{T} \in S$, then, $T(f(\bar{x}), f(S)) \cap(-\epsilon q-D \backslash\{0\}) \neq \emptyset$, for all $\epsilon>0$, and (ii) in Theorem 4.10 is false. In fact, for any $\beta>0$ and $d \in T(f(\bar{x}), f(S)) \cap(-\epsilon q-$ $D+\beta U) \subset-\operatorname{int} R_{+}^{2}, T^{2}(f(\bar{x}), f(S), d)=T^{\prime \prime}(f(\bar{x}), f(S), d)=R^{2}$, since $f(\bar{x}) \in \operatorname{int}(f(S))$. But $\bar{x} \in \operatorname{LPAE}(f, S, \epsilon)$. This implies that the conditions of Theorem 4.10 are not necessary.

Example 4.15. Let $D=R_{+}^{2}, S=\left\{\left(x_{1}, x_{2}\right) \in R^{2}: x_{2} \geq\left|x_{1}\right|\right\}, f: S \rightarrow R^{2}, f\left(x_{1}, x_{2}\right)=\left(x_{1}, x_{2}\right)^{T}, q=$ $(1,1)^{T}$ and $\epsilon=1$. We consider $\bar{x}=\epsilon q \in S$. It is easy to see that $\bar{x} \in \operatorname{LAE}(f, S, \epsilon q)=\operatorname{AE}(f, S, \epsilon q)$. But $T(f(\bar{x}), f(S)) \cap(-\epsilon q-D)=\left\{\left(y_{1}, y_{2}\right)^{T} \in R^{2}: y_{1} \leq-1, y_{2} \leq-1, y_{2} \geq y_{1}\right\} \neq \emptyset$, and the 
condition (ii) of Theorem 4.7 is false. In fact, if we take $d=(-2,-2)^{T} \in T(f(\bar{x}), f(S)) \cap$ $(-\epsilon q-D)$, then, $d^{\perp}=\left\{\left(y_{1}, y_{2}\right)^{T}: y_{1}+y_{2}=0\right\},-$ cl cone $(D+\epsilon q+d)=R^{2}$ and $T^{\prime \prime}(f(S), f(\bar{x}), d)=$ $\left\{\left(y_{1}, y_{2}\right)^{T} \in R^{2}: y_{2} \geq y_{1}\right\}$. Therefore, $T^{\prime \prime}(f(S), f(\bar{x}), v) \cap v^{T} \cap(-$ cl cone $(D+\epsilon q+v))=\left\{\left(y_{1}, y_{2}\right) \in\right.$ $\left.R^{2}: y_{1}+y_{2}=0, y_{1} \leq 0\right\}$.

Example 4.16. Let $D=R_{+}^{2}, S=\left\{\left(x_{1}, x_{2}\right) \in R^{2}: x_{2}=\left|x_{1}\right|^{3 / 2}\right\}, f: S \rightarrow R^{2}, f\left(x_{1}, x_{2}\right)=\left(x_{1}, x_{2}\right)^{T}$, $q=(1,1)^{T}$, and $\epsilon>0$. We consider $\bar{x}=(0,0)^{T} \in S$. It is easy to see that $T(f(\bar{x}), f(S)) \cap(-\epsilon q-$ $D)=\emptyset$ and $f(S)-f(\bar{x}) \subset T(f(\bar{x}), f(S))$. That is, the condition (i) of Theorem 4.7 is valid, and $\bar{x} \in \operatorname{LPAE}(f, S, \epsilon q)=\operatorname{PAE}(f, S, \epsilon q)$, for all $\epsilon>0$.

Theorem 4.17. Let $\bar{x} \in S, \epsilon \geq 0$ and $D=R_{+}^{m}$.

(i) If $f_{-}^{\prime}(\bar{x}, d) \cap\left(-\epsilon q-\operatorname{int} R_{+}^{m}\right)=\emptyset$, for any unit vector $d \in T(\bar{x}, S)$, then $\bar{x} \in \operatorname{LWAE}(f, S, \epsilon q)$.

(ii) If $f_{-}^{\prime}(\bar{x}, d) \cap\left(-\epsilon q-R_{+}^{m} \backslash\{0\}\right)=\emptyset$, for any unit vector $d \in T(\bar{x}, S)$, then $\bar{x} \in \operatorname{LAE}(f, S, e q)$.

Where, $f_{-}^{\prime}(\bar{x}, d)=\left(\left(f_{-}^{\prime}\right)_{1}(\bar{x}, d), \ldots,\left(f_{-}^{\prime}\right)_{m}(\bar{x}, d)\right)^{T}$.

Proof. (i) Suppose, on the contrary, that $\bar{x} \notin \operatorname{LWAE}(f, S, \epsilon q)$, then, there exists $x_{k} \in S \backslash\{\bar{x}\}$, $k \in N$ and $x_{k} \rightarrow \bar{x}$ such that $f\left(x_{k}\right)-f(\bar{x}) \in-\epsilon q-\operatorname{int} R_{+}^{m}$. Let $d_{k}=\left(x_{k}-\bar{x}\right) /\left\|x_{k}-\bar{x}\right\|$ and $t_{k}=\left\|x_{k}-\bar{x}\right\|$, then, $t_{k} \rightarrow 0, d_{k} \rightarrow d \in T(\bar{x}, S)$ and $\|d\|=1$. Hence,

$$
\frac{f\left(x_{k}\right)-f(\bar{x})}{t_{k}}=\frac{f\left(\bar{x}+t_{k} d_{k}\right)-f(\bar{x})}{t_{k}} \in-\epsilon q-\operatorname{int} R_{+}^{m}-\left(\frac{1}{t_{k}}-1\right) \epsilon q .
$$

Since $t_{k} \downarrow 0$, there exists $k_{1} \in N$ such that $\left(f\left(x_{k}\right)-f(\bar{x})\right) / t_{k} \in-\epsilon q-\operatorname{int} R_{+}^{m}$, for all $k \geq k_{1}$. Hence,

$$
\frac{f_{i}\left(x_{k}\right)-f_{i}(\bar{x})}{t_{k}}+\epsilon q_{i}<0, \quad \forall i \in\{1, \ldots, m\}, k \geq k_{1} .
$$

Therefore,

$$
\begin{aligned}
\left(f_{-}^{\prime}\right)_{i}(\bar{x}, d)+\epsilon q_{i} & =\liminf _{t \downarrow 0} \frac{f_{i}(\bar{x}+t h)-f_{i}(\bar{x})}{t}+\epsilon q_{i} \\
& \leq \liminf _{n \rightarrow \infty} \frac{f_{i}\left(\bar{x}+t_{n} d_{n}\right)-f_{i}(\bar{x})}{t_{n}}+\epsilon q_{i}<0, \quad \forall i \in\{1, \ldots, m\} .
\end{aligned}
$$

Which is a contradictions to the assumption. This completes the proof.

(ii) Similar to the proof of (i), we have there exists $x_{k} \in S \backslash\{\bar{x}\}, k \in N$ and $x_{k} \rightarrow \bar{x}$ such that $f\left(x_{k}\right)-f(\bar{x}) \in-\epsilon q-R_{+}^{m} \backslash\{0\}$. Hence, there exists $k_{1} \in N$ such that $\left(f\left(x_{k}\right)-f(\bar{x})\right) / t_{k} \in$ $-\epsilon q-R_{+}^{m} \backslash\{0\}$, for all $k \geq k_{1}$. It is easy to see that, if we take an appropriate subsequences $x_{n}^{k}$ 
and $t_{n}^{k}$ of $x_{k}$ and $t_{k}$, respectively, then there exist an index $i_{0} \in\{1, \ldots, m\}, n_{0} \in N$ and $k_{0} \in N$ such that

$$
\begin{gathered}
\frac{f_{i}\left(x_{n}^{k}\right)-f_{i}(\bar{x})}{t_{n}^{k}}+\epsilon q_{i} \leq 0, \quad \forall i \in\{1, \ldots, m\}, \quad \forall k \geq k_{0}, n \geq n_{0}, \\
\frac{f_{i_{0}}\left(x_{n}^{k}\right)-f_{i_{0}}(\bar{x})}{t_{n}^{k}}+\epsilon q_{i_{0}}<0, \quad \forall k \geq k_{0}, n \geq n_{0} .
\end{gathered}
$$

Therefore, $\left(f_{-}^{\prime}\right)_{i}(\bar{x}, d)+\epsilon q_{i} \leq 0$, for all $i \in\{1, \ldots, m\}$, and $\left(f_{-}^{\prime}\right)_{i_{0}}(\bar{x}, d)+\epsilon q_{i_{0}}<0$, which is a contradiction to the assumption. This completes the proof.

Remark 4.18. The following necessary conditions for $\epsilon$-local weakly (efficient) solutions may not be true.

$$
\begin{array}{cl}
\bar{x} \in \operatorname{LWAE}(f, S, \epsilon q) \Longrightarrow f_{-}^{\prime}(\bar{x}, d) \cap\left(-\epsilon q-\operatorname{int} R_{+}^{m}\right)=\emptyset, & \forall d \in T(\bar{x}, S) . \\
\bar{x} \in \operatorname{LAE}(f, S, \epsilon q) \Longrightarrow f_{-}^{\prime}(\bar{x}, d) \cap\left(-\epsilon q-R_{+}^{m} \backslash\{0\}\right)=\emptyset, & \forall d \in T(\bar{x}, S) .
\end{array}
$$

See the following example.

Example 4.19. Let $f(x)=\left(f_{1}(x), f_{2}(x)\right)^{T}: R \rightarrow R^{2}$,

$$
f_{1}(x)= \begin{cases}x \sin \frac{1}{x}, & x \neq 0, \\ 0, & x=0,\end{cases}
$$

$f_{2}(x)=x, \epsilon=2 / \pi, q=(1,1)^{T}, S=\{x \in R:-2 / \pi \leq x \leq 2 / \pi\}$. Consider the following problem:

$$
\min _{x \in S} f(x)
$$

It is easy to see that $\bar{x}=0$ is an $\epsilon q$-efficient solution of (MP), but, $\left\{d \in R:\left(f_{-}^{\prime}(\bar{x}, d)+\epsilon q\right) \in\right.$ - int $\left.R_{+}^{2}\right\} \cap T(\bar{x}, S) \neq \emptyset$. In fact,

$$
\left(f_{1}\right)_{-}^{\prime}(\bar{x}, d)=\liminf _{t \downarrow 0} \frac{f_{1}(t h)-f_{1}(0)}{t}=\liminf _{t \downarrow 0} h \sin \frac{1}{t h}=-|d|, \quad \forall d \in R .
$$

$\left(f_{2}\right)_{-}^{\prime}(\bar{x}, d)=d$, for all $d \in R$. It is obvious that $-1 \in\left\{d \in R:\left(f_{-}^{\prime}(\bar{x}, d)+\epsilon q\right) \in-\right.$ int $\left.R_{+}^{2}\right\}$. On the other hand, $T(\bar{x}, S)=R$. Hence, $\left\{d \in R:\left(f_{-}^{\prime}(\bar{x}, d)+\epsilon q\right) \in-\operatorname{int} R_{+}^{2}\right\} \cap T(\bar{x}, S) \neq \emptyset$. 


\section{Acknowledgments}

This work was partially supported by the National Science Foundation of China (no. 10771228 and 10831009), the Research Committee of The Hong Kong Polytechnic University, the Doctoral Foundation of Chongqing Normal University (no.10XLB015) and the Natural Science Foundation project of CQ CSTC (no. CSTC. 2010BB2090).

\section{References}

[1] K. Miettinen and M. M. Mäkelä, "On cone characterizations of weak, proper and Pareto optimality in multiobjective optimization," Mathematical Methods of Operations Research, vol. 53, no. 2, pp. 233-245, 2001.

[2] L. G. Huang and S. Y. Liu, "Cone characterizations of Pareto, weak and proper efficient points," Journal of Systems Science and Mathematical Sciences, vol. 23, no. 4, pp. 452-460, 2003 (Chinese).

[3] A. Engau and M. M. Wiecek, "Cone characterizations of approximate solutions in real vector optimization," Journal of Optimization Theory and Applications, vol. 134, no. 3, pp. 499-513, 2007.

[4] B. Aghezzaf and M. Hachimi, "Second-order optimality conditions in multiobjective optimization problems," Journal of Optimization Theory and Applications, vol. 102, no. 1, pp. 37-50, 1999.

[5] A. Cambini, L. Martein, and M. Vlach, "Second-order tangent sets and optimaity conditions," Tech. Rep., Japan Advanced Studies of Science and Technology, Hokuriku, Japan, 1997.

[6] J.-P. Penot, "Second-order conditions for optimization problems with constraints," SIAM Journal on Control and Optimization, vol. 37, no. 1, pp. 303-318, 1999.

[7] B. Jiménez and V. Novo, "Optimality conditions in differentiable vector optimization via second-order tangent sets," Applied Mathematics and Optimization, vol. 49, no. 2, pp. 123-144, 2004.

[8] C. Gutiérrez, B. Jiménez, and V. Novo, "New second-order directional derivative and optimality conditions in scalar and vector optimization," Journal of Optimization Theory and Applications, vol. 142, no. 1, pp. 85-106, 2009.

[9] G. Bigi, "On sufficient second order optimality conditions in multiobjective optimization," Mathematical Methods of Operations Research, vol. 63, no. 1, pp. 77-85, 2006.

[10] S. S. Kutateladze, "Convex E-programming," Soviet Mathematics. Doklady, vol. 20, pp. 390-1393, 1979.

[11] I. Vályi, "Approximate saddle-point theorems in vector optimization," Journal of Optimization Theory and Applications, vol. 55, no. 3, pp. 435-448, 1987.

[12] J.-C. Liu, " $\varepsilon$-properly efficient solutions to nondifferentiable multiobjective programming problems," Applied Mathematics, vol. 12, no. 6, pp. 109-113, 1999.

[13] S. Bolintinéanu, "Vector variational principles; $\varepsilon$-efficiency and scalar stationarity," Journal of Convex Analysis, vol. 8, no. 1, pp. 71-85, 2001.

[14] J. Dutta and V. Vetrivel, "On approximate minima in vector optimization," Numerical Functional Analysis and Optimization, vol. 22, no. 7-8, pp. 845-859, 2001.

[15] A. Göpfert, H. Riahi, C. Tammer, and C. Zălinescu, Variational Methods in Partially Ordered Spaces, Springer, New York, NY, USA, 2003.

[16] E. M. Bednarczuk and M. J. Przybyła, "The vector-valued variational principle in Banach spaces ordered by cones with nonempty interiors," SIAM Journal on Optimization, vol. 18, no. 3, pp. 907-913, 2007.

[17] G. Chen, X. Huang, and X. Yang, Vector Optimization. Set-Valued and Variational Analysis, vol. 541 of Lecture Notes in Economics and Mathematical Systems, Springer, Berlin, Germany, 2005.

[18] D. Gupta and A. Mehra, "Two types of approximate saddle points," Numerical Functional Analysis and Optimization, vol. 29, no. 5-6, pp. 532-550, 2008.

[19] C. Gutiérrez, B. Jiménez, and V. Novo, "A Set-valued ekeland's variational principle in vector optimization," SIAM Journal on Control and Optimization, vol. 47, no. 2, pp. 883-903, 2008.

[20] C. Gutiérrez, R. López, and V. Novo, "Generalized $\varepsilon$-quasi-solutions in multiobjective optimization problems: existence results and optimality conditions," Nonlinear Analysis: Theory, Methods $\mathcal{E}$ Applications, vol. 72, no. 11, pp. 4331-4346, 2010.

[21] D. J. White, "Epsilon efficiency," Journal of Optimization Theory and Applications, vol. 49, no. 2, pp. 319-337, 1986.

[22] S. Helbig, "One new concept for $\varepsilon$-efficency," talk at Optimization Days, Montreal, Canada, 1992.

[23] T. Tanaka, "A new approach to approximation of solutions in vector optimization problems," in 
Proceedings of APORS, M. Fushimi and K. Tone, Eds., vol. 1995, pp. 497-504, World Scientific, Singapore, 1994.

[24] Y. Sawaragi, H. Nakayama, and T. Tanino, Theory of Multiobjective Optimization, vol. 176 of Mathematics in Science and Engineering, Academic Press, Orlando, Fla, USA, 1985.

[25] W. D. Rong and Y. Ma, " $\varepsilon$-properly efficient solution of vector optimization problems with set-valued maps," OR Transaction, vol. 4, pp. 21-32, 2000.

[26] J. Borwein, "Proper efficient points for maximizations with respect to cones," SIAM Journal on Control and Optimization, vol. 15, no. 1, pp. 57-63, 1977.

[27] L. R. Huang, "Separate necessary and sufficient conditions for the local minimum of a function," Journal of Optimization Theory and Applications, vol. 125, no. 1, pp. 241-246, 2005.

[28] W. Rudin, Functional Analysis, McGraw-Hill Series in Higher Mathematics, McGraw-Hill, New York, NY, USA, 1973. 